This is a post-peer-review, pre-copyedit version of an article published in European Journal of Information Systems. The definitive publisher-authenticated version [Macredie, RD and Mijinyawa, K (2011), "A theory-grounded framework of Open Source Software adoption in SMEs", European Journal of Informations Systems, 20(2), 237-250] is available online at: http://www.palgrave-journals.com/ejis/journal/v20/n2/abs/ejis201060a.html.

\title{
A Theory-Grounded Framework of Open Source Software Adoption in SMEs
}

Robert D Macredie and Kabiru Mijinyawa, School of Information Systems, Computing and Mathematics, Brunel University

\begin{abstract}
The increasing popularity and use of Open Source Software has led to significant interest from research communities and enterprise practitioners, notably in the small business sector where this type of software offers particular benefits given the financial and human capital constraints faced. However, there has been little focus on developing valid frameworks that enable critical evaluation and common understanding of factors influencing Open Source Software adoption. This paper seeks to address this shortcoming by presenting a theory-grounded framework for exploring these factors and explaining their influence on Open Source Software adoption, with the context of study being small to medium sized Information Technology businesses in the UK. The framework has implications for this type of business - and, we will suggest, more widely - as a frame of reference for understanding, and as tool for evaluating benefits and challenges in, Open Source Software adoption. It also offers researchers a structured way of investigating adoption issues and a base from which to develop models of Open Source Software adoption. The study reported in this paper used the Decomposed Theory of Planned Behaviour as a basis for the research propositions, with the aim of: (i) developing a framework of empirical factors that influence Open Source Software adoption; and (ii) appraising it through case study evaluation with 10 UK Small to Medium Sized Enterprises in the Information Technology sector. The demonstration of the capabilities of the framework suggests that it is able to provide a reliable explanation of the complex and subjective factors that influence attitudes, subjective norms and control over the use of Open Source Software. The paper further argues that the Decomposed Theory of Planned Behaviour proved useful in this research area and that it can provide a variety of situation-specific insights related to factors that influence the adoption of Open Source Software.
\end{abstract}

Keywords: Open Source Software (OSS); Small to Medium Sized Enterprises (SMEs); adoption; innovation; Decomposed Theory of Planned Behaviour; case study evaluation.

\section{Introduction}

Open Source Software (OSS) is increasingly popular and widely used across different types and sizes of enterprise. Briefly, OSS is software where the license model grants individuals, groups, and organizations extensive rights to use, modify, and redistribute the binary and source-code of the original and modified/derived works, without requiring license royalty-fees (OSD version 1.9; Fitzgerald 2004; Wheeler 2007). The availability and openness of OSS source-code enables communities of users, developers, sponsors, and scholars 
to independently develop, re-design, and validate OSS, improving its functionality, value and quality and making it freely available to others. In contrast, proprietary and closed-source software - including variations such as 'evaluation/trial software', 'shareware', and 'freeware' - can be described as software that is available only in its binary form (i.e., not in a form that can be easily modified), that generally requires the payment of license fees by enterprises/users, and that legally restricts user rights and vendor liabilities (Dedrick and West 2004; Larsen et al. 2004; Mindel et al. 2007).

The growth in adoption, and rise in use, of OSS is made clear in a range of recent reports. For example, a periodic survey published by Netcraft (2010) reports that the use of Apache (a popular OSS web server) grew by 47.26\% over the last four years, up from 53,287,298 in March 2006 to 112,747,166 in March 2010. The Eclipse Foundation (2009) also published a survey suggesting that the proportion of computers running Linux (an open source desktop/workstation operating system) grew from $20 \%$ to $27 \%$ between 2007 and 2009. The same survey also reported that Linux's use as server/deployment environment grew from $37 \%$ to $43 \%$ in the same period. A regional survey of top smart-phone platforms suggests that the Google-developed Android OSS platform has seen the proportion of phones running it as the core system rising 5.2\% in just four months; up from $3.8 \%$ in November 2009 to 9\% in February 2010 (comScore MobiLens Service 2010). These examples highlight the rapidly expanding adoption of OSS and illustrate the diverse areas in which OSS is now used. Studies report OSS also being used in the public sector (Valimaki et al. 2005), healthcare (Fitzgerald and Kenny 2003), large enterprises (Dedrick and West 2004; Overby et al. 2006) and developing economies (Kshetri 2004; Mindel et al. 2007).

The potential spread of OSS into ever more application areas makes it important for businesses and researchers to understand the factors influencing decisions about its use, and its adoption in particular settings. However, research into OSS adoption is still in its infancy (Dedrick and West 2003; Fitzgerald and Kenny 2003; Holck et al. 2005; Larsen et al. 2004), and there has been little research focused on the development of valid frameworks that help us to understand and evaluate issues influencing adoption. Though such frameworks of OSS adoption would be useful to all businesses, there are contexts where decision-making related to OSS adoption is likely to be more critical. One such context, which forms the focus of this paper, is that of Small to Medium Sized Enterprises (SMEs), which have significant financial and human capital constraints that affect decision-making around software adoption in ways not generally encountered by larger enterprises (Gelinas and Bigras 2004), which are likely to have both greater financial resources and greater in-house expertise in relation to Information and Communications Technologies (ICTs). Studying adoption in the more constrained SME context is by its nature, we suggest, more likely to give insights into a greater number of factors that influence adoption - and that these insights may be used to inform a better understanding of OSS adoption in general. This leads to the broad question behind the research reported in this paper: what factors influence the adoption of OSS in SMES and why?

In response, this paper presents a theory-grounded framework for exploring and explaining factors that influence the adoption of OSS within SMEs. The empirical framework that emerges is likely to be useful to IS researchers seeking to undertake a comparative analysis of factors influencing general OSS adoption, developing a similar 
framework of ICT adoption, or developing reliable measures of factors influencing the adoption of OSS in enterprises. From a practice perspective, the framework provides an empirical frame of reference of issues influencing SMEs' adoption of OSS which may be applied as a valid underlying framework for the strategic adoption of OSS in the enterprise.

The remainder of this paper is divided into six sections. In the next section, relevant literature will be analysed to show that there is a lack of valid factors and theories in the area and to set out the implications for exploring and understanding the adoption of OSS. This leads to our proposed approach to developing a theory-grounded framework, followed by the selection of the Decomposed Theory of Planned Behaviour (DTPB) as the most suitable adoption theory for this study. The paper then presents the operationalisation of the DTPB, where we justify and define the roles of its constructs for exploring and understanding SMEs' adoption of OSS. The subsequent section will present the design of a case-based research study, which applied the research framework as a tool for the empirical observation and analysis of data on the adoption of OSS across 10 UK SMEs in the Information Technology sector. The paper then presents the outcomes of case sampling and data analysis, showing that the empirical factors were developed from rich and diverse sources of evidence. This leads to a discussion of the empirical findings with respect to the application of the research framework for conclusion drawing. Through this, we will also demonstrate the exploratory and explanatory capabilities of the empirical framework as a theory of OSS adoption. The implications of the findings will be discussed from research and practice perspectives, before reflecting on the limitations of the framework and suggesting future research. The final section will present concluding remarks about the research presented in this paper.

\section{Framing OSS Adoption in Theory}

Existing literature suggests that the factors influencing OSS adoption are complex and subjective (Dedrick and West 2003; Fitzgerald 2004; Forrester Consulting 2007; Giera 2004) and that there is a lack of valid and generalisable frameworks that help researchers and practitioners to better understand and model OSS adoption. One reason may well be the relative newness of the area, noted by authors such as Fitzgerald and Kenny (2003), Holck et al. (2005) and Larsen et al. (2004). In line with early research on general ICT adoption, studies of OSS have used semantic contexts of technology, organisation and environment to discuss the factors influencing adoption. Although such contexts enable semantic analysis of ICT adoption (Kuan and Chau 2001; Overby et al. 2006), the use of reliable and widely-accepted theories with established construct validity, generalisability and better exploratory and explanatory capabilities (Miles and Huberman 1994; Yin 2003) is likely to be more suitable for developing a theoretically-valid and -reliable conceptual model and an empirical framework of OSS adoption. Thus, we suggest that it is worth looking at whether existing theories of general ICT adoption can be applied to provide an underlying theoretical framework for exploring, explaining and achieving a common understanding of factors that influence the adoption of OSS.

The relative paucity of existing research and related frameworks of OSS adoption leads to a currently limited understanding of the factors that play a part in adoption (Holck et al. 2005; Larsen et al. 2004; Overby et al. 2006). This creates difficulties in conducting a critical, comparative analysis to better understand and validate 
empirical findings; in this study, we sought to augment the limited understanding of OSS adoption with knowledge of similar factors from literature in other areas of ICT adoption. An aim of this approach was to develop valid definitions of existing and emerging adoption factors which could subsequently be applied in the development of reliable measures of factors influencing the adoption of OSS.

The arguments made so far provide justification for using a theory-grounded approach to explore adoption factors, frame valid explanations of them and develop a framework that can provide a better understanding of the factors and their influence on OSS adoption. However, the diversity of existing ICT adoption models and theories - such as the Theory of Reasoned Action (TRA) (Albarracin et al. 2001; Fishbein and Ajzen 1975), the Theory of Planned Behaviour ('pure' TPB) (Ajzen 1985; Ajzen 1991), the Technology Acceptance Model (TAM) (Davis 1989; Venkatesh and Davis 2000) and the Decomposed Theory of Planned Behaviour (DTPB) (Liker and Sindi 1997; Shih and Fang 2004; Taylor and Todd 1995a) - made it important to identify the theory that was likely to be most suitable for this research study. The evaluation of models and theories conducted as part of the study led to the choice of the DTPB for two major reasons. First, the DTPB has three belief components (attitude, subjective norms, and perceived behavioural control) which are applicable to a wide variety of complex and subjective factors associated with ICT adoption (Benbasat and Zmud 1999; Taylor and Todd 1995a,b), and therefore relevant for exploring and developing valid explanations of diverse factors influencing the adoption of OSS. Second, the belief components within the DTPB are decomposed into their belief structures, which provides greater scope for identifying complex factors than that offered by other theories and models such as the TRA and the traditional TPB, which have monolithic belief components (Taylor and Todd 1995b; Venkatesh et al. 2003). The two reasons for choosing DTPB outlined above also reflect the important exploratory and explanatory capability of the DTPB - capabilities that allow us to achieve high construct validity (Shih and Fang 2004; Taylor and Todd 1995a) in our analysis of factors affecting OSS adoption.

\section{Understanding OSS Adoption Using the DTPB}

Having discussed the reasons behind this study's use of the DTPB, this section demonstrates its operationalisation within the context of OSS adoption in SMEs, leading to the research conceptual framework. The generic constructs of the DTPB and their nomological networks have been extensively discussed in the literature (see, for example, Ajzen 1991; Fishbein and Ajzen 1975; Liker and Sindi 1997; Shih and Fang 2004; Taylor and Todd 1995a,b). The belief structures within the DTPB provide structures within which factors influencing adoption may be identified. Accordingly, we focused primarily on the eight belief structures of the DTPB and their individual roles in enabling the identification and definition of empirical factors and explanations of their influence. The belief structures are: (1) relative advantage, (2) complexity and (3) compatibility (attitudinal belief structures); (4) peer influences and (5) superior influences (normative belief structures); and (6) self-efficacy, (7) resource facilitating conditions and (8) technology facilitating conditions (perceived behavioural control structures). These structures, and their value in identifying factors relevant to the adoption and use of OSS, will now be discussed in turn. A position drawn from analysis of relevant literature will be presented in relation to whether each construct was likely to have a positive or negative influence on 
OSS adoption in SMEs. This helped inform the conceptual framework's development prior to the case study work.

\section{Attitudinal Belief Structures}

The attitudinal belief structures - relative advantage, complexity and compatibility - are used to identify and explain respondents' perceptions of whether the use of OSS is favourable or unfavourable in the SME (Ajzen 1991; Davis 1989; Fishbein and Ajzen 1975).

The first construct, relative advantage, identifies and explains the degree to which using OSS is perceived to provide benefits which supersede those of its precursor (Benbasat and Moore 1992; Rogers 1995; Taylor and Todd 1995a). Such perceptions are related to economic benefits (see, for example, cost saving in Fitzgerald 2004; Giera 2004), convenience benefits (see, for example, trialability in Dedrick and West 2004; Rogers 1983), satisfaction benefits (see, for example, quality characteristics in Fitzgerald 2004; Overby et al. 2006), and image enhancements and performance (Taylor and Todd 1995a,b; Venkatesh et al. 2003). The nature of these features, the role of relative advantage and its influence on the 'attitude' construct, suggest that relative advantage factors will have a positive influence on attitude towards the use of an OSS. This also sets the scope of using relative advantage to explore benefits, drivers and innovation-related motivations for the use of an OSS.

Complexity is the second construct; it identifies and explains the degree to which an OSS is perceived to be difficult to understand, learn or use (Rogers 1995; Taylor and Todd 1995a). Such perceptions of difficulty appear to be associated with issues such as 'lack of support', as reported in, for example, Giera (2004), and Wang and Wang (2001). The nature of these features of complexity, the role of complexity and its influence on the 'attitude' construct, suggest that complexity factors will have a negative influence on attitude towards the use of an OSS. The 'complexity' construct may be used in an exploratory way and is suitable for exploring innovation-related risks and challenges in using an OSS.

The third construct is compatibility, which identifies and explains the extent to which an OSS is perceived to fit with the adopter's existing values and software policies, previous experiences and relevant skills, or current IT needs (Benbasat and Moore 1992; Rogers 1995; Taylor and Todd 1995a,b)). An example is of perceptions related to functionality (Fitzgerald and Kenny 2003; Forrester Consulting 2007; Overby et al. 2006), which was identified to fit with the feature of 'current IT needs' and can be argued to fit with 'existing values'. The relevance of the features of compatibility, and its role and influence on adopter-attitude suggests that compatibility factors will have a positive influence on attitude towards their use of OSS.

\section{Normative Belief Structures}

The normative beliefs structures (or subjective norms) - peer influences and superior influences - are used to identify and explain the influence of different referent groups on perceptions of whether the use of OSS is good or bad in the SME (Ajzen 1991; Fishbein and Ajzen 1975).

The fourth construct, peer influences, is concerned with the perception that peers, such as friends, families, and colleagues or other external actors, influence the normative beliefs of decision makers associated with whether 
using an OSS is good or bad (Ajzen 1991; Taylor and Todd 1995). An example is the influence exerted by family members in the case of a family-owned SME (Houghton et al. 2001). The different members of a referent group, their subjective influence and the role of 'peer influences' as a construct, suggest that peer influence factors will have a subjective influence on the normative beliefs of decision makers about the use of an OSS.

The fifth construct, superior influences, relates to perceptions formed as a result of information from secondary sources, such as the Internet, TV and printed media. The suggestion is that such information can influence the normative beliefs of decision makers associated with OSS use (Ajzen 1991; Fishbein and Ajzen 1975; Taylor and Todd 1995). The information sources associated with 'superior influence' is analogous to the influence of the 'communication channel' discussed in Rogers' (1995) innovation diffusion theory (IDT), seeing, for example, the role of the Internet as a key information and communication infrastructure supporting the spread of OSS products and support services. The diverse sources of information and range of communication channels, and the role of such 'superior influences', suggest that the factors associated with them will have a subjective influence on normative beliefs held by decision makers about the use of an OSS, and that it should be possible to identify such secondary information sources.

\section{Perceived Behavioural Control Belief Structures}

The 'control' belief structures, of which there are three, allow the identification and explanation of perceptions of control over the personal/internal or external factors that may facilitate or constrain the use of OSS by a SME (Ajzen 1991; Taylor and Todd 1995). The roles of these last three constructs in exploring factors of control over the use of OSS will now be discussed.

Self-efficacy, the sixth construct, identifies and explains the personal/internal ability or confidence that an individual has to use an OSS successfully (Taylor and Todd 1995b). Factors identified in the literature that fit with this construct include OSS innovativeness (Dedrick and West 2003). The features of self-efficacy and its role in the control over usage suggest that self-efficacy enabling factors will have a positive influence on an individual's perceived control over the use of an OSS system.

Resource facilitating conditions, the seventh construct, identifies and explains the relevance of supporting resources, such as time and money, that may facilitate or constrain the use of OSS (Taylor and Todd 1995b). This construct fits with factors such as IT capital investment, which has been reported as an essential resource for the development of SMEs' IT capacity and capability (Kwan and West 2005; Mannaert and Ven 2005). The lack of such resources may inhibit the use of OSS suggesting that the resource facilitating condition construct is relevant for exploring associated factors and that having relevant resource facilitating conditions will have a positive influence, while a lack of them will have a negative influence on control over the use of OSS.

The final construct in the framework relates to technology facilitating conditions, used to identify and explain technology compatibility issues that may facilitate or constrain the use of an OSS system (Taylor and Todd 1995b). Some researchers have suggested that access to relevant 'IT infrastructure', such as computers, network hardware, and Internet connection, are important technology compatibility issues in the adoption of OSS 
(Mehrtens et al. 2001). As another essential condition, a lack of technology facilitating conditions may inhibit the use of OSS in the organisation. Thus, exploring technology facilitating conditions is relevant to this study, since it seems that having relevant technology facilitating conditions will have a positive influence, while a lack of them will have a negative influence, on control over the use of OSS.

\section{Method and Case Sample}

In this study, we applied empirical research paradigms that fit with the research question, the need to identify factors and understand their influence, and the aim to develop a theory from that understanding. This led to our unit of analysis, factors influencing the adoption of OSS, and to us taking an interpretivist epistemological stance owing to the view that factors influencing the adoption of OSS are likely to be complex and subjective across different SMEs. With this subjectivist view, we sought to observe, understand factors and to explain their influence on the adoption of OSS, leading us to a qualitative research mode (Eisenhardt 1989; Miles and Huberman 1994). Owing to various issues, including the contemporary nature and the relative newness of this research area, and the need to generalise empirical findings to a theory about the adoption of OSS, we chose to apply a case study strategy (Miles and Huberman 1994; Yin 2003) to explore the use of OSS across multiple SMEs.

Data analysis using this strategy was implemented in two key stages (as illustrated in Figure 1): within-case, to the identify and categorize factors from individual study cases (Gable 1994; Yin 1994); and cross-case, to collate similar factors, triangulate their associated evidence, and develop definitions of factors rich in features and high in content validity (Eisenhardt 1989; Mayring 2007). Figure 1 also shows the different roles of the theoretical framework as an analysis tool (Eisenhardt 1989; Patton 1999; Yin 1994): the framework was used to identify initial theoretical categories; applied in conducting credibility checks during data interpretation, dependability checks in data categorisation, and consistency checks when defining factors; and applied in the interpretation of within-case results and cross-case results. The use of a theoretical framework was part of the design approach implemented to ensure the structured, methodological design of data collection, analysis, and reporting.

The data collection included demographic information from the participating organisations' web-sites and indepth interviews about issues influencing the decisions to use OSS. We developed open- and close-ended interview questions based on the previously discussed theoretical framework (Eisenhardt 1989; Hoepfl 1997; Miles and Huberman 1994). For example, we asked questions related to attitudes towards the use of OSS, including the relative advantage and benefits of use, the areas of use, the external actors playing a part in decisions to use, and the skills and resources supporting use of OSS. Per-case transcripts were developed from the audio-recordings of the interview sessions. The interview data provided rich, comparative information on the SME contexts and issues influencing the decisions related to OSS use in the participating organisations.

Most of the interview participants were managers and senior staff in the SMEs studies. Individuals in such positions are seen as change agents and decision makers (Martin 2005; Taylor and Murphy 2004), and they have the authority to provide insights on the use of OSS in their organisation, as well as being able to identify other 
sources of information. All 10 case organisations (C01-C10; where C07 was a maximum-variation sample and a non-adopter of OSS) were IT SMEs in the UK, and this common sampling criteria provided scope for comparison and triangulation in the multiple-case data analysis. The diversity of the sampled cases allowed us to observe complexity and subjectivity in the factors influencing the adoption of OSS across the different organisations. For example, the organisations used different OSS that fitted with their business needs and specialties. The OSS consultants (C01, C03, C06, and C08), software developers (C04, C07, C09, and C10), and embedded system developers (C02 and C05) used diverse OSS including workstations (Debian, Ubuntu, and Suse), servers (Apache, LAMP stack, and Tomcat), databases (PostgreSQL, MySQL), email and browser applications (Thunderbird, Firefox, and Kmail), and other office and systems applications (Easy-CMS, IPTables, OpenOffice, OpenSSL, OpenVPN, and PhP).

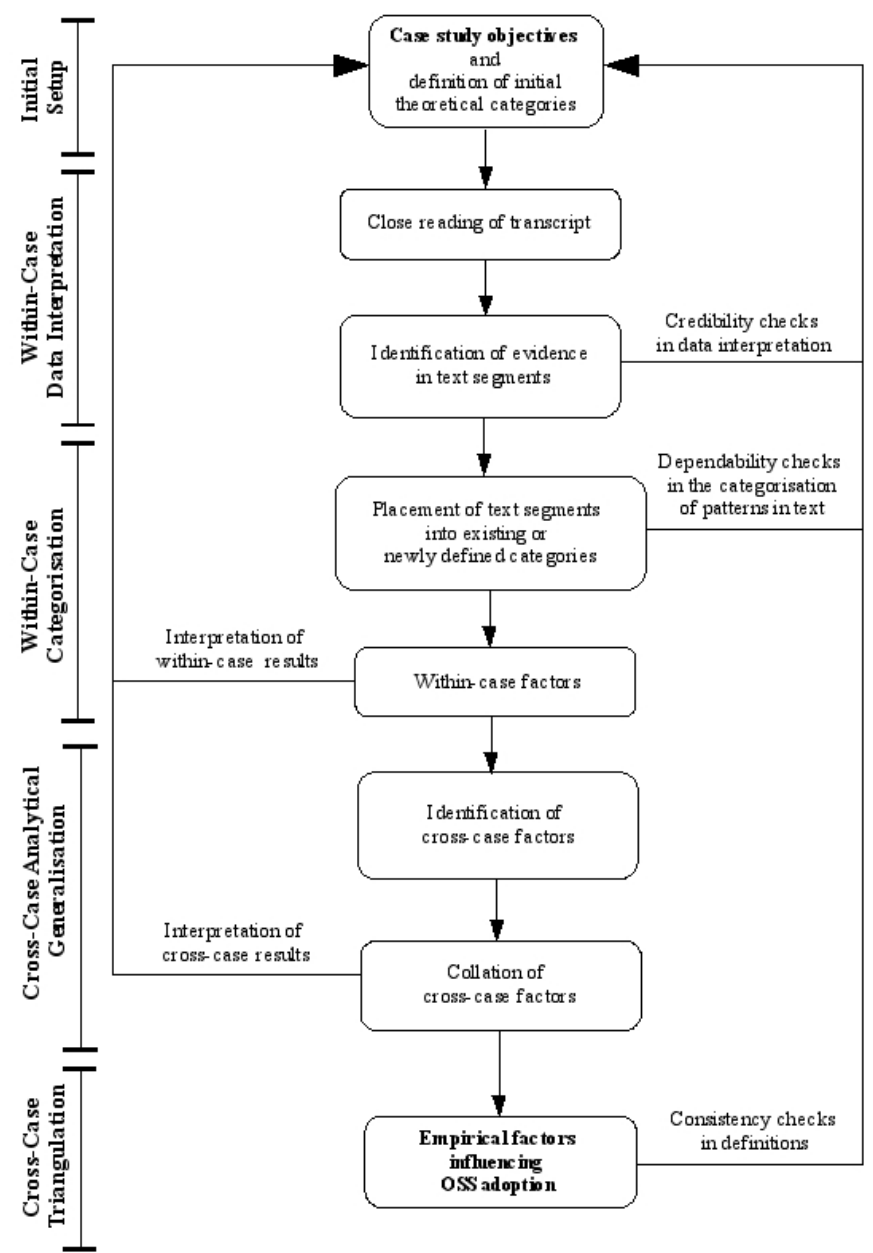

Figure 1. Data Analysis Model

\section{Data Analysis}

The within-case and cross-case analysis of data led to the identification and theorisation of 16 factors influencing the SMEs in this study. The factors identified (across the eight theoretical constructs of the framework) include license cost-saving (relative advantage), lack of drivers (complexity), functionality 
(compatibility), support community (peer influences), web media (superior influences), innovativeness (selfefficacy), capital investment (resource facilitating conditions), and Internet infrastructure (technology facilitating conditions).

These factors, their analytical features, and associated theoretical constructs are summarized in Table 1. The theorisation and validation of these factors was based on the triangulation of evidence (Mayring 2007; Miles and Huberman 1994; Sandelowski 2003) from at least four cases, as illustrated in the frequency of triangulation (Table 2) and a comparative chart in the relative triangulation of the factors (Figure 2). For example, the theorisation of 'license cost-saving' was supported by the triangulation of evidence from cases $\mathrm{C} 01, \mathrm{C} 02, \mathrm{C} 04$, C05, C06, and C08 (see illustration in Tables 1, 2 and 3); reliability, from cases C01, C03, C06, and C08; and hardware compatibility, from cases C04, C06, C08, and C09. Table 3 shows that features of the factor 'license cost-saving' were identified in the evidence from representative cases and collated in the emergent description of the factor. This process of theory-building is known to enhance the development of reliable factors (Eisenhardt 1989; Gable 1994) that are rich in features, from the triangulation of evidence from multiple sources. 
Table 1. Summary of the Analysis of Influencing Factors

\begin{tabular}{|c|c|c|c|c|}
\hline Factors & Contexts & Features & Constructs & Related Cases \\
\hline \multirow{3}{*}{$\begin{array}{l}\text { License cost- } \\
\text { saving }\end{array}$} & Public licensing & Economic benefit & Relative advantage & $\mathrm{C} 01$ and $\mathrm{C} 05$ \\
\hline & Royalty-free licenses & & & $\mathrm{C} 01, \mathrm{C} 02, \mathrm{C} 04, \mathrm{C} 06$, and $\mathrm{C} 08$ \\
\hline & Scalable licensing & & & $\mathrm{CO} 2$ and $\mathrm{C0} 4$ \\
\hline \multirow[t]{2}{*}{ Lack of drivers } & Inaccessible software specifications & Difficult to use & Complexity & $\mathrm{C} 01, \mathrm{C} 02, \mathrm{C} 03$, and $\mathrm{C} 09$ \\
\hline & $\begin{array}{l}\text { Poor support from hardware } \\
\text { manufacturers }\end{array}$ & & & $\mathrm{C} 01, \mathrm{C} 06, \mathrm{C} 08$, and $\mathrm{C} 09$ \\
\hline \multirow[t]{3}{*}{ Functionality } & Embedded systems & $\begin{array}{l}\text { existing values and } \\
\text { current IT needs }\end{array}$ & Compatibility & $\mathrm{C} 05$ and $\mathrm{C} 09$ \\
\hline & Office information processing & & & $\begin{array}{l}\mathrm{C} 01, \mathrm{C} 02, \mathrm{C} 03, \mathrm{C} 04, \mathrm{C} 05, \mathrm{C} 09 \text {, } \\
\text { and } \mathrm{C} 10\end{array}$ \\
\hline & Software development & & & $\mathrm{C} 04$ and $\mathrm{C} 05$ \\
\hline \multirow{4}{*}{$\begin{array}{l}\text { Support } \\
\text { community }\end{array}$} & developer-developer & Peer relationships & Peer influences & $\mathrm{C} 01$ and $\mathrm{C} 05$ \\
\hline & user-developer & & & $\mathrm{C} 01, \mathrm{C} 04, \mathrm{C} 05$, and $\mathrm{C06}$ \\
\hline & user-user & & & $\mathrm{C} 0,1 \mathrm{C} 05$, and $\mathrm{C} 09$ \\
\hline & vendors distribute OSS & & & $\mathrm{C} 01$ and $\mathrm{C} 04$ \\
\hline \multirow[t]{2}{*}{ Web media } & Distribution medium & $\begin{array}{l}\text { Information and } \\
\text { communication }\end{array}$ & Superior influences & $\mathrm{C03}, \mathrm{C} 05$, and $\mathrm{C} 09$ \\
\hline & Forums and mailing-lists & channels & & $\mathrm{C} 03, \mathrm{C} 05, \mathrm{C} 09$, and $\mathrm{C} 10$ \\
\hline \multirow[t]{3}{*}{ Innovativeness } & Creativeness & Confidence & Self-efficacy & $\mathrm{C} 02, \mathrm{C} 06$, and $\mathrm{C} 09$ \\
\hline & Freedom of choice & & & $\mathrm{C} 02$ and $\mathrm{C} 05$ \\
\hline & Open mindedness & & & $\mathrm{C} 02, \mathrm{C} 05, \mathrm{C} 06$, and $\mathrm{C} 09$ \\
\hline \multirow[t]{2}{*}{$\begin{array}{l}\text { Capital } \\
\text { investment }\end{array}$} & External support & Money and time & $\begin{array}{l}\text { Resource facilitating } \\
\text { conditions }\end{array}$ & $\mathrm{C} 01$ and $\mathrm{C} 05$ \\
\hline & Staff training & & & $\mathrm{C} 03, \mathrm{C} 08$, and $\mathrm{C} 09$ \\
\hline \multirow[t]{2}{*}{$\begin{array}{l}\text { Internet } \\
\text { infrastructure }\end{array}$} & Internet connectivity & $\begin{array}{l}\text { Technology } \\
\text { compatibility }\end{array}$ & $\begin{array}{l}\text { Technology } \\
\text { facilitating conditions }\end{array}$ & $\mathrm{C} 03, \mathrm{C} 05, \mathrm{C} 09$, and $\mathrm{C} 10$ \\
\hline & Network bandwidth & & & $\mathrm{C} 03$ \\
\hline
\end{tabular}


Table 2. Frequency of the Triangulation of Factors

\begin{tabular}{lc|cccc}
\hline Factors & & \multicolumn{2}{c}{ Frequency of Triangulation } & \\
& Cumulative Sources & Context-1 & Context-2 & Context-3 & Context-4 \\
\hline License cost-saving & 6 & 2 & 5 & 2 & NA \\
\hline Lack of drivers & 6 & 4 & 4 & NA & NA \\
\hline Functionality & 7 & 2 & 7 & 3 & NA \\
\hline Support community & 5 & 2 & 4 & NA & NA \\
\hline Web media & 5 & 3 & 2 & 4 & NA \\
\hline Innovativeness & 4 & 3 & 3 & NA & NA \\
\hline Capital investment & 5 & 2 & 1 & NA & NA \\
\hline Internet infrastructure & 4 & 4 & & & 2 \\
\hline
\end{tabular}

Table 3. Triangulation of Evidence of License Cost-Saving

\begin{tabular}{|c|c|}
\hline Description & The economic benefits of using open source including public licensing, royalty-free licenses, and scalable licensing. \\
\hline Features & Public licensing (C01, C05); Royalty-free licenses (C01,C02, C04, C06, C08); Scalable licensing (C02, C04) \\
\hline \multirow[t]{6}{*}{$\begin{array}{l}\text { Data } \\
\text { Extracts }\end{array}$} & $\begin{array}{l}\text { If I need a program to do something, I can just do a quick search, download it, and install it [in] a matter of seconds... I don't } \\
\text { have to worry about licenses[, open source are] free for those who are prepared to make the effort to understand it to use it } \\
\text { properly... I think the license is obviously an advantage. No licensing cost - C01, 2007-10-25. }\end{array}$ \\
\hline & $\begin{array}{l}\text { Licensing cost are a big issue, depending on the open source software you are talking about... There's definitely a cost } \\
\text { advantage [and] that generally occurs when [you] need to deploy [multiple copies] of a piece of software[, but the costs] of } \\
\text { the closed-source competition aren't really good for that particular application - C02, 2007-11-19. }\end{array}$ \\
\hline & $\begin{array}{l}\text { [There is a] huge benefit from a licensing point of view [but] probably not as big as most people would think... I am not } \\
\text { having to pay for licenses for each and every test server that I am setting up - C04, 2007-11-13. }\end{array}$ \\
\hline & $\begin{array}{l}\text { I've never paid for Linux... its free. That's a distinction feature... Having registered with Ubuntu for the desktop [using] } \\
\text { Debian, [it just happens and] a little icon comes up when there is a new upgrade. [This] happens with Windows as well. But } \\
\text { with Windows, you have to have paid MS first[, and] proprietary operating systems [are] very private, and sequestered, and } \\
\text { exclusive, and you have to pay for access - C05, 2007-11-14. }\end{array}$ \\
\hline & $\begin{array}{l}{[\mathrm{I}] \mathrm{t} \text { beats me why people pay for commercial web engines when eighty percent of the Internet is driven by Apache... [T]his }} \\
\text { is a benefit that I can pass onto customers - C06, 2007-11-21. }\end{array}$ \\
\hline & $\begin{array}{l}\text { I think the main advantage is partly[, ] its cost and flexibility. But its also the ability to bring more value to a project... } \\
\text { [W]ith any commercial off-the-shelf software, if the software doesn't do what the projects needs it to do, then I need to buy } \\
\text { some other software, or work around the problem - C08, 2007-11-13. }\end{array}$ \\
\hline
\end{tabular}




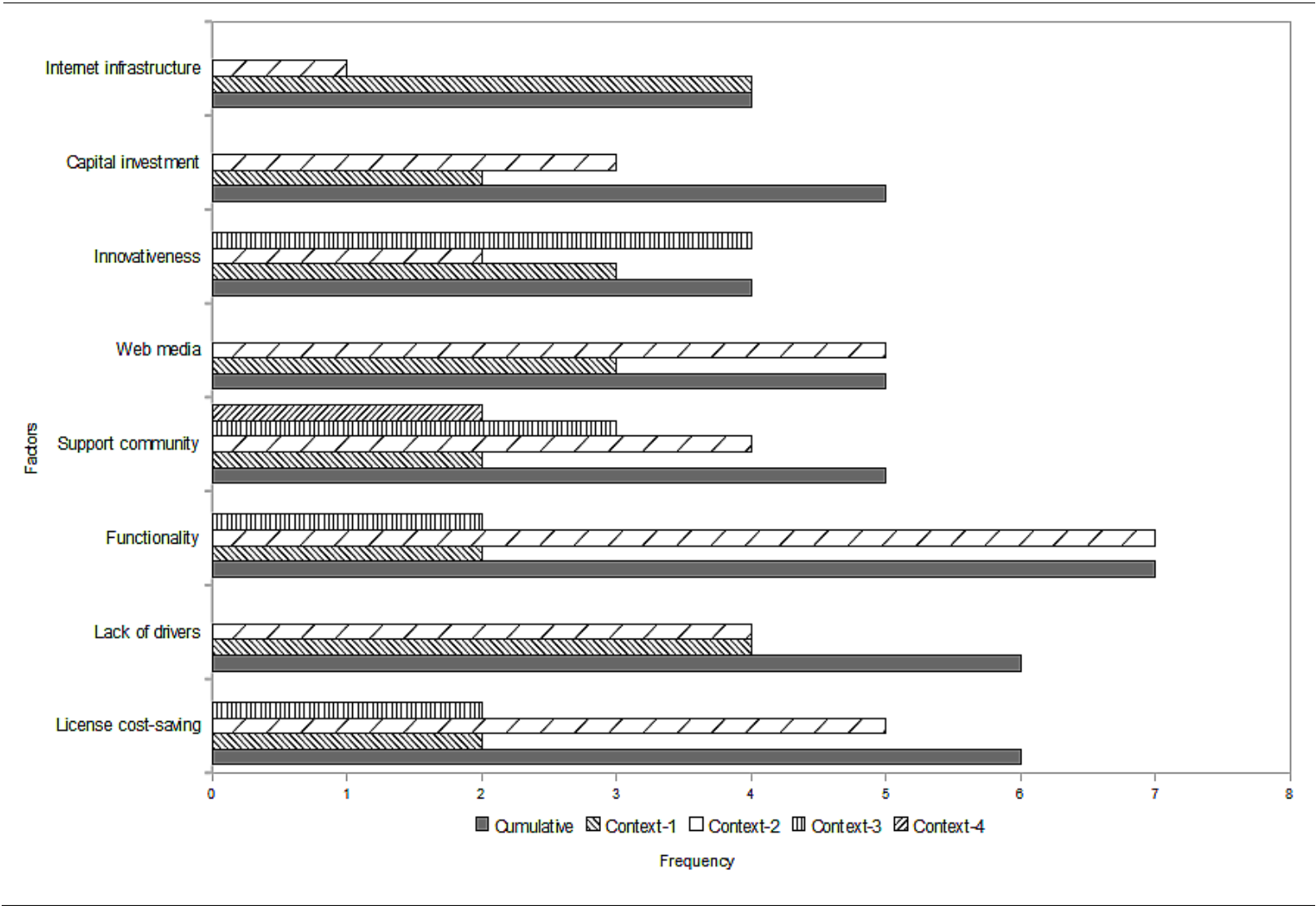

Figure 2. Relative Triangulation of the Factors

\section{Findings}

The factors identified as a result of the data analysis help in framing answers to the research question set-out earlier in this paper. We present the factors in an empirical framework of OSS adoption in SMEs - see Figure 3. The framework shows the factors associated with each belief structure from the DTPB. The belief structures enabled valid identification and categorisation of the factors and their nomological interrelationships. The belief components, the intention, and the behaviour constructs allowed us to explain the influence of the factors on the adoption of OSS. The exploratory and explanatory capability of the framework will now be demonstrated to show that it provides a valid explanation of the factors and their influence on the adoption of OSS. In doing so, the illustrative factors from each of the eight belief structures in Figure 3 will be explained. No particular method was applied in the selection of the factors used for this illustration and other factors associated with the same theoretical construct can be explained in a similar way, taking into consideration the association of the factors with the features of the belief structures.

\section{License Cost-Saving}

The first illustrative factor in Figure 3 is license cost-saving, defined as the degree of economic benefit gained from using OSS, including public licensing, royalty-free licenses, and scalable licensing. These three features of license cost-saving were observed in different contexts. The features highlight the economic attractiveness to decision makers in the SMEs of using OSS, and this can be most significant for multiple and large scale installations. For instance, one manager noted: “there's definitely a cost advantage [and] that generally occurs 
when [you] need to deploy [many] copies of a piece of software [, and] the pricing structure of the closedsource competition aren't really good for that particular application” (C02, 2007-11-19). The features of public licensing and royalty-free licensing make 'innovativeness' more affordable in lowering the costs of creativity, giving all innovators equal opportunity and freedom to use OSS and source-code, and the choice of supporting technologies.

The license cost-saving from using OSS may be a characteristic economic benefit, consistent with criteria of the OSS license model (OSD version 1.9) including royalty-free and unlimited distribution of OSS binaries and source code. We share the view that license cost-saving is seen as a motivating factor and an economic benefit in the use of OSS (Holck et al. 2004; Larsen et al. 2004; Valimaki et al. 2005). Such economic benefits are likely to be of value to small businesses looking to leverage their limited IT budgets and scarce resources in the adoption of ICT (Darch and Lucas 2002; Martin and Matlay 2003). While there can be other cost issues owing to the complexity of open source products and related services (Giera 2004; Larsen et al. 2004), we believe that license cost-saving is a valuable economic benefit for resource-constrained IT adopters.

\section{Lack of Drivers}

The second illustrative factor from Figure 3 is 'lack of drivers', a complexity factor operationalised as a difficulty in using OSS owing to a lack of software specifications and hardware-support from manufacturers. In this context, one manager noted that "a lot of the manufacturers won't release specifications to the OSS community to write proper drivers. So a lot of it had to be reverse engineered" (C01, 2007-10-25). However, this issue might be most related to desktop platforms: "[lack of drivers has been] a big issue on the desktop [...] the fact that the major PC hardware suppliers, AMD and Intel [having] very good relationship with [the] Open Source community for a long time helps things a lot" (C10, 2007-11-26). Because this factor can inhibit functionality in using OSS under unsupported hardware, it highlights the importance of participation and collaboration in OSS communities to address gaps not filled by the original hardware manufacturers, and therefore signifies the importance and significant contribution made by the user and developer communities.

There appears to be little discussion of the lack of hardware driver support under OSS platforms. However, studies do suggest that support for OSS adoption is important (Dedrick and West 2004; Fitzgerald and Kenny 2003; Giera 2004; Wang and Wang 2001), and support for hardware drivers can be seen as a related issue in this context. Managers and other practitioners in SMEs may need to consider the availability of support for hardware drivers for their existing and future hardware relevant to their adoption of OSS. 


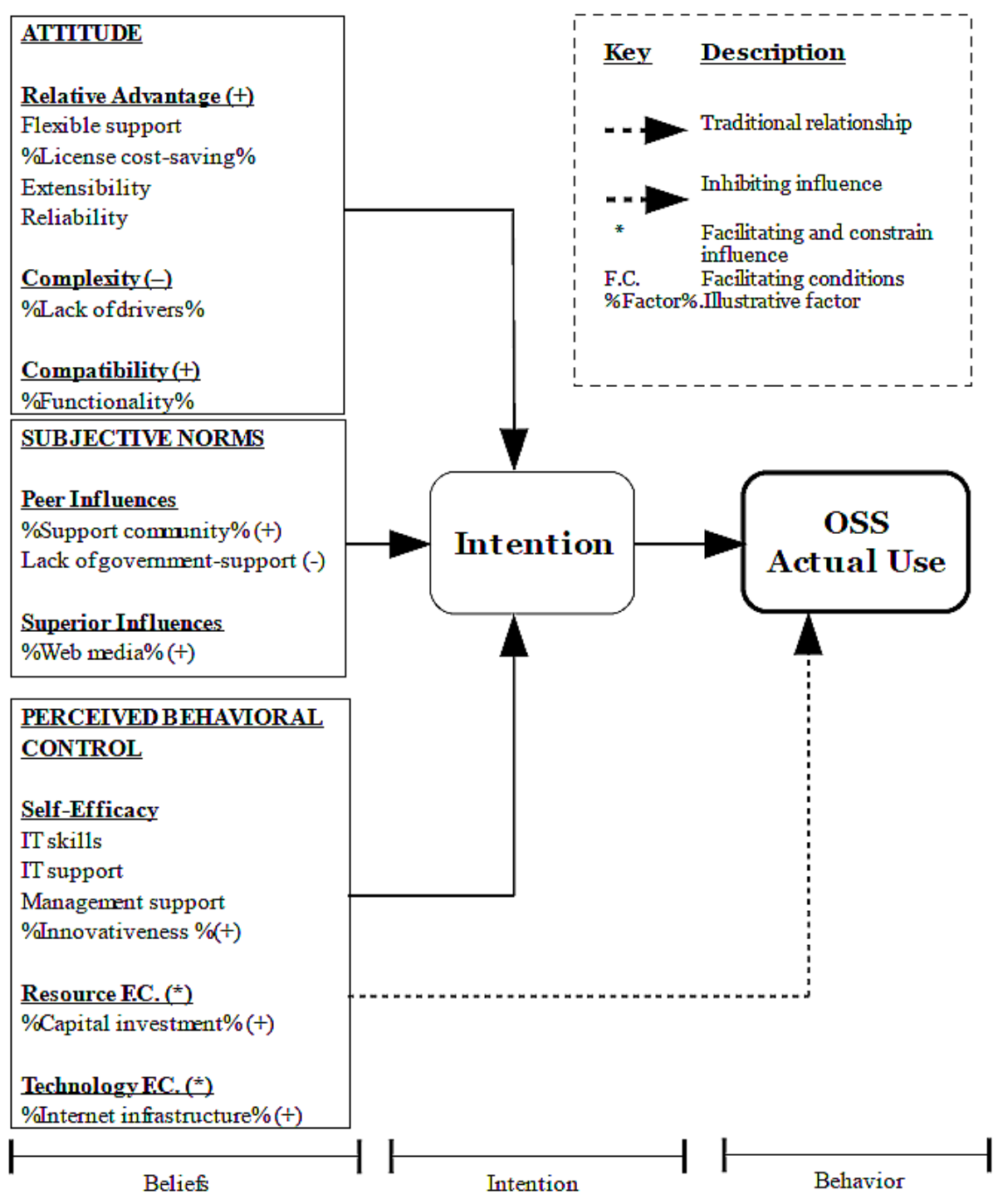

Figure 3. A Framework of Open Source Software Adoption in SMEs

\section{Functionality}

Functionality, the third illustrative factor in Figure 3, was operationalised as the compatibility of OSS with adopters' IT needs, including office information processing, software development, and embedded systems. These forms of the factor were seen as important IT needs of the participants and therefore make functionality a compatibility factor. For instance, one manager noted that, "for most offices, for most functions, you need to browse the web, read emails, and create documents and spreadsheets, if you like. So, between Firefox, Thunderbird and OpenOffice, all of which run on MS, as well as Linux, you can port your organisation onto those three fairly painlessly over time" (C01, 2007-10-25). This observation is consistent with the view that OSS provides a flexible software development base, and also fits with general office information system needs (Fitzgerald 2004; Glynn et al. 2005). The diverse areas of functionality offered makes OSS a versatile facilitator 
of innovation, providing individuals, groups, and organisations with diverse information technology and business tools to support their innovation. The flexibility of extending the functionality of OSS allows innovators to have an open mind about the techniques and tools available to them.

However, we found that an individual's perceptions of functionality associated with software can be subjective, affected by the quality of its user-interface: “occasionally, for [desktop] software, sometimes the interfacing isn't as good in Open Source. But, for majority of our uses, that's not really an issue" (C02, 2007-11-19). Given the range of OSS application types, the quality of user-interface is likely to be mainly relevant for end-user applications on desktop platforms, helping to explain why the quality of OSS functionality may be perceived as being variable across desktop, server, and embedded platforms.

As illustrated in Figure 3, the factors associated with these attitudinal belief structures lead to attitudes about the use of OSS that influence the intention to use or not to use OSS. Both flexible support (a relative advantage) and functionality (a compatibility factor) have a positive influence on attitude towards use. Conversely, lack of hardware drivers, as a complexity factor, has a negative influence on attitudes towards use.

\section{Support Community}

The next belief component in Figure 3 is subjective norms, encompassing the fourth factor of 'support community', identified in the forms of open participation and shared support. These forms encompass the role of peers within the OSS communities, who can influence other people with respect to OSS use. In this context, one manager stated that, "you need to be able to connect to a community of like-minded users who will help you out when you get stuck [...] I am saying that intellectual curiosity and caring about what goes on behind the system are a characteristics of open source people" (C05, 2007-11-14). Thus, we can operationalise support community as peer influences in OSS communities that promote open participation and shared support.

This factor has a positive influence on the normative beliefs of decision makers related to the use of OSS, positively contributing to intention to use. The OSS support community is global, diverse and dynamic, and there continues to be 'investment' of human and capital resources that allows communities to develop, grow and support their users, developers and innovators. This identifies the need for continued human and capital 'investment' through participation in, and collaboration and contribution to, OSS communities. OSS communities are consistently reported as being important sources of technical and informational support, through the provision of free and commercial support services, enabling use of OSS by individuals and organisations (Giera 2004; Overby et al. 2006; Wang and Wang 2001).

\section{Web Media}

'Web media' is the fifth factor illustrated in Figure 3, operationalised as superior influences of the web as a source and medium of information relevant to the development and distribution of OSS. For instance, one manager noted, "realistically, Internet based information is where most information is going to come from; most people that are interested in OSS get most of their information from the Internet" (C03, 2007-11-28). However, other evidence suggests that there might be limitations in the availability of OSS documentation: "its very often, installation or compilation instructions are very brief or non-existent. The more widely supported and more 
widely used things like MySQL, like OpenOffice are pretty well documented" (C06, 2007-11-21). This statement highlights the diversity of quality and availability of software support and documentation for OSS.

Web media are a source of OSS products and documentation, and the common platform for interaction between OSS users and developers who use, develop and maintain OSS and its supporting communities. Studies also suggests that 'web media' is an important adoption enabler and represents a primary domain for the OSS products, information and services provided by OSS projects and communities (Giera 2004; Overby et al. 2006; Yamauchi et al. 2000). Thus, web media, in terms of content and services, is an important factor in the adoption of OSS and may be the backbone for the global, diverse and dynamic OSS phenomenon. This factor fits as a secondary source of information; this means it is a superior influence factor, and its context has a positive influence on the normative beliefs of decision makers related to the use of OSS. Similarly, this influence positively contributes to intention to use.

\section{Innovativeness}

The sixth factor is innovativeness in the forms of open-mindedness, creativity and self-motivation. These forms of innovativeness fit with the self-efficacy feature of confidence, leading us to operationalise this factor as the confidence (in terms of open-mindedness, creativity, and self-motivation) of an individual to use OSS. One context of this factor, as experienced by one SME manager is that, "the major skill is the ability to go out and find things that solve your problem" (C09, 2007-11-23). Innovativeness in the forms of creativeness and freedom of choice in using OSS are, we believe, partly enabled by the flexibility of public and royalty-free licensing of OSS; these features allow everyone equal opportunity to use, modify and re-design OSS applications and source-code in their own ways.

In the contexts discussed, and the illustration in Figure 3, innovativeness is a self-efficacy construct that has a positive influence on control over use of OSS, and this positively contributes to the intention to use. SMEs are often seen as innovative organisations (Gelinas and Bigras 2004; Martin 2005; Taylor and Murphy 2004), and this willingness and confidence to innovate encourages decision makers within them to consider the technological, legal and business factors relevant to their adoption of OSS (Holck et al. 2004).

\section{Capital Investment}

Capital investment is the seventh illustrative factor; it was identified as a resource facilitating condition and operationalised as the money and staff-time resources needed to facilitate the use of OSS. In these contexts, one manager noted, "if you don't have [the] expertise yourself or the time to develop that expertise, then you need to hire-in" (C01, 2007-10-25). We believe that capital investment is relevant for providing adequate computing and 'network infrastructure' to support communication and other business activities. Studies have consistently shown money to be an essential resource for the development of IT capability, achieved through hiring-in or training staff to support ICT adoption in SMEs (Houghton et al. 2001; Taylor and Murphy 2004), and through consultancy on OSS product assessment, configuration, integration and maintenance (Holck et al. 2004; Kumar and Krishnan 2005). 
Time was also identified as being essential for developing OSS expertise and managing the transition to new OSS platforms: "Often there is a little bit more involved on a Linux side in understanding what goes on [...] it is a bit more difficult to, for example, set-up a web server on Linux, [and] to administer firewall" (C08, 2007-1113). As an essential facilitating condition, capital investment has a positive influence on control over the use of OSS and this positively contributes to the intention to use.

\section{Internet Infrastructure}

The last illustrative factor in Figure 3, internet infrastructure, was identified as a technology facilitating condition operationalised as Internet-connectivity and -bandwidth facilitating the use of OSS. This factor is consistent with the earlier discussion of web media. Many participants noted the need for adequate networking infrastructure to support low- and high-bandwidth activities, including email, instant messaging and forum services and support, which may be unavailable as standard across all Internet service providers. For instance, one manager stated that, "I would never have started using it [a specific piece of OSS] actively if it wasn't for the Internet because I won't have been able to get a copy of it. Um so yeah, without the Internet, I don't think OSS could exist in its current form" (C03, 2007-11-28). As suggested in the discussion on 'capital investment', providing staff with adequate Internet infrastructure may require investment in network-services. However, depending on the scale of network bandwidth requirements, quality of service, availability of Internet service providers, and government legislation regarding Internet services, the required investment in Internet infrastructure can be subjective for staff across different work settings.

This reinforces the view of the Internet as essential infrastructure in the contemporary information age (Houghton et al. 2001; Kuan and Chau 2001; Martin 2005; Mehrtens et al. 2001), and reflects the importance of IT hardware to facilitate the use of OSS (Holck et al. 2004; Larsen et al. 2004). This factor has a positive influence on control over the use of OSS and also contributes to intentions to use. Managers in SMEs may need to consider the availability of relevant IT infrastructure including Internet-connectivity and -bandwidth in their adoption of OSS.

The DTPB posits that a lack of facilitating conditions can inhibit actual use. In the context of SME adoption of OSS, a consideration of essential resources and technology compatibility issues is particularly important because, as illustrated in Figure 3, a lack of facilitating conditions (such as capital investment and Internet infrastructure) can inhibit OSS adoption. While managers and other decision-makers are generally concerned about the investment of scarce resources for ICT adoption (Glynn et al. 2005; Kwan and West 2005), including access to suitable network-bandwidth and the quality of Internet services, having suitable infrastructure is likely to specifically influence the success or failure of their use of OSS.

\section{Non-adoption}

Interestingly, the analysis of data from case C07 suggests there may be issues common to the adoption of OSS and closed-source software; these issues provided insight into the non-adoption of OSS in case C07. For example, the participant in case C07 said that: "we are basically a MS partner, so we can get good deals with that" (C07, 2007-10)-25. This experience corresponds to an economic benefit of license cost-saving as a result 
of the 'good deals' received from the software vendor. Another example is that connectivity to a support community was also relevant in case $\mathrm{C} 07$, and the participant noted: "there's a Microsoft Development Network [...] There are forums which people can give suggestion to MS and stuff like that really" (C07, 2007-10-25). This view highlights the need for Internet infrastructure and web media for connecting to, and participating in, the relevant internet community (the MSDN). These views suggests that license 'deals' and access to the support community (via web media and using internet infrastructure) contributed to the non-adoption of OSS in case 07.

\section{Implications for Research and Practice}

The theory-grounded framework for understanding factors that influence the adoption of OSS in SMEs has two important implications. First, the framework provides a frame of reference (Agarwal 2000) for understanding factors and their influence on the adoption of OSS. For instance, researchers may find the operational definitions of the theoretical constructs and the illustrative factors useful as theory-grounded analysis tools for understanding and explaining factors influencing the adoption of OSS. Second, the generalisability of the framework makes it directly usable for undertaking a critical and comparative analysis to validate and better understand factors and their influence on OSS adoption. One example in this context is the operational definitions; the features of the factors and the associated theoretical constructs illustrated could be used in developing hypotheses and propositions to validate the roles and the interrelationships between these concepts. In our experience, a lack of well-defined factors and theoretical concepts has been a problematic issue in conducting comparative analysis of factors to validate empirical findings. Another example is that the factors' features could be applied in developing measures of the factors influencing the adoption of OSS.

From a practice perspective, there are three immediate implications. First, the framework provides a frame of reference for practitioners seeking to develop deep insight (Agarwal 2000) and common understanding of factors that influence OSS adoption. This is consistent with the view that frameworks are useful for evaluators and practitioners seeking to model the adoption of OSS (see, for example, the discussions in Benbasat and Moore 1992; and Dedrick and West 2003).

Second, owing to the strong exploratory and explanatory capability of the underlying theory (the DTPB), the framework allows the development of a broad view of, and insights into, the factors influencing the adoption of OSS in SMEs. The framework is directly usable and therefore has a strong practical relevance (see, for discussion, Agarwal 2000; and Benbasat and Zmud 1999); managers and other practitioners could apply the framework to better identify and understand diverse contexts of attitudinal, normative and controllability factors and to target situation-specific factors in their adoption of OSS.

Thirdly, the framework provides strong justification for courses of action (Benbasat and Moore 1992; Dedrick and West 2003) in the adoption of OSS. For example, managers and other decision-makers could use the arguments and empirical insights about influencing factors, such as license cost-saving, functionality and innovativeness, as justification for considering OSS as a viable IT solution, and for raising staff awareness about the benefits, challenges and business opportunities arising from the use of OSS. This would encourage the 
evaluation of situation-specific factors that might play a part in the attitudes towards the use of OSS and the investment of human and capital resources.

Finally, the value of the underpinning theory merits comment. The use of the DTPB in the study, the diverse factors identified and the strong explanations derived show that the DTPB is well-suited to exploring and understanding factors and explaining their influence on the adoption of OSS by SMEs. Many studies have applied the pure TPB and the DTPB and achieved useful research findings (see, for example, Benbasat and Zmud 1999; Liker and Sindi 1997; Shih and Fang 2004; Taylor and Todd 1995a,b; Venkatesh et al. 2003), and the introduction of the DTPB to the area of OSS adoption -we believe this study to be among the first - extends the scope of ICT adoption models and theories applicable to the study of OSS adoption.

\section{Limitations and Future Research}

A limitation of this study stems from the use of maturing qualitative research methodologies (Eisenhardt 1989). This is relevant to the quality of the research design, the rigour in the data collection (including managing the high volume of qualitative data), the data analysis and the credibility of the findings. These issues may explain unseen participant- and researcher-bias in the data analysis, leading to less-than-perfect understanding of alternative, corroborating or opposing information and insights related to influencing factors. In order to minimize the effects of this limitation, we applied recommended measures including using a case study database and protocol to maintain an audit trail of case data and analysis processes (Lincoln and Guba 1985; Patton 1999). In so doing, we are confident that the applied research methods are credible, and the analysis and related findings are acceptably valid and relevant to the research problem and objectives set-out in the paper.

Despite our confidence in the methods and findings, we acknowledge that the research area of OSS adoption is relatively new and the framework presented in this paper has its own limitations. First, the scope of factors presented in the paper is limited owing to the sample of workplace setting (SMEs in the IT industry), the heterogeneity of the observed OSS applications and their areas of use, and the number of study cases. We acknowledge that other influencing factors - such as user legal rights, unnecessary fears, and risk expectancy (Overby et al. 2006; Wheeler 2007); government IT policies, vendor support, and industry monopoly (Lin 2006; Mindel et al. 2007; Wheeler 2007); and product knowledge and internal resources (Dedrick and West 2003; Glynn et al. 2005; Overby et al. 2006) - may be prevalent in other organisations and industries. This limitation in the sampling scope is common to interpretive and qualitative studies (Dedrick and West 2004; Eisenhardt 1989; Mayring 2007) and invites future research to extend the scope of factors influencing the adoption of OSS. Researchers undertaking such studies could apply a similar theory-building approach and exploratory research design, drawing on the work presented in this paper and taking advantage of the underlying theory as an analysis tool.

Again common to qualitative studies, the theoretical generalisability of the framework remains to be established across a wide population. However, we argue that the framework has analytical generalisability owing to the generalisability of the DTPB (Shih and Fang 2004; Taylor and Todd 1995a,b; Venkatesh et al. 2003) and the use of a theory-building approach in identifying/developing the influencing factors. Future research may seek to 
ascertain the theoretical generalisability of the framework using a confirmatory study that tests the validity and reliability of the framework across a wider population of OSS adopters - either limited to SMEs or for other organisational types. Such tests may provide decision-makers and researchers with useful benchmarks about theoretical constructs and factors which may be closely related to the success and failure of the adoption of OSS. The tests could yield useful insights into the roles and influence of the theoretical constructs and influencing factors. For example, the tests could seek to explore: (1) whether the framework might be adequately valid and reliable for predicting adoption of OSS; (2) what theoretical constructs might be most relevant in understanding the adoption of OSS; (3) which factors might be significant in the adoption of OSS; and (4) whether the influence of the benefits and challenges of using OSS are significant enough to attract the interests of new adopters, stimulating a broader scale adoption of OSS. The questions could be posed for SMEs, as a direct extension of the work reported in this paper, and/or for other organisational types or for specific sectors of industry, commerce or public service.

The study propositions for these tests would be based on the theoretical constructs and factors presented in the framework. For example, the following propositions from the framework could be tested in future studies: (a) having flexible support has a significant and positive influence on attitudes towards using OSS; (b) extensibility will have a significant and positive influence on attitudes towards using OSS; (c) the support community has a significant and positive influence on the normative beliefs of decision makers about using OSS; and (d) IT support has a significant and positive influence on control over the use of OSS. The outcomes of such studies would contribute to the accumulation of valid and reliable measures and scales for evaluating theoretical concepts and factors influencing the adoption of OSS.

\section{Conclusions}

This paper has presented a theory-grounded framework that is of value in helping to better explore, explain and understand the adoption of OSS in SMEs. The lack of valid factors and reliable theories that enable better exploration and understanding of factors and their influence on OSS adoption by SMEs was discussed, leading to the proposal and justification of a theory-grounded approach to addressing this research gap. We selected and operationalised the DTPB for this purpose and presented our research methodology, discussing the key reasons for applying an exploratory, qualitative, multiple case study research design. The analysis of data that led to the identification of empirical factors and the development of a framework of OSS adoption in SMEs was also discussed. The analysis of data has led to the identification of feature-rich factors and the development of, we argue, a valid and generalisable framework of OSS adoption in SMEs that we contend is useful for research and practice. Other implications of the framework were also discussed from research and practice perspectives, before noting its limitations and making suggestions for future research to extend its analytical capabilities and validate its generalisability across a broader population of OSS adopters. 


\section{References}

AGARWAL R (2000) Individual acceptance of information technologies. In Framing the Domains of let Management: Projecting the Future Through the Past (ZMUD RW Ed). Cincinnati, OH: Pinnacle Education Resources, 85-104.

AJZEN I (1991) Theory of planned behaviour. Organisational Behavior and Human Decision Processes 50, $179-211$.

BENBASAT I and MOORE GC (1992) Development of measures for studying emerging technologies. Proceedings of the Twenty-Fifth Hawaii International Conference on Systems Science (HICSS) 4, 315-324.

BENBASAT I and ZMUD RW (1999) Empirical research in Information Systems: the practice of relevance. MIS Quarterly 23(1), 3-16.

\section{COMSCORE MOBILENS SERVICE}

http://www.comscore.com/Press_Events/Press_Releases/2010/4/comScore_Reports_February_2010_U.S._ Mobile_Subscriber_Market_Share (23-June-2010).

DAVIS FD (1989) Perceived usefulness, perceived ease of use, and user acceptance of information technology. MIS Quarterly 13(3), 319-339.

DEDRICK J and WEST J (2003) Why firms adopt Open Source platforms: a grounded theory of innovation and standards adoption, Standard Making: a Critical Research Frontier for Information Systems. MISQ Special Issue Workshop, Seattle, December 12-14. 236-257.

DEDRICK J and WEST J (2004) An exploratory study into Open Source platform adoption. Proceedings of the 37th Hawaii International conference on Systems Sciences, 1-10.

ECLIPSE FOUNDATION (2009) A survey of the Eclipse user community which supports both open source and proprietary software environments. http://arstechnica.com/open-source/news/2009/05/eclipse-surveyresults-show-growth-in-linux-open-source.ars (23-June-2010).

EISENHARDT KM (1989) Building theories from case study research. Academy of Management Review 14(4), 532-550.

FISHBEIN M and AJZEN I (1975) Belief, Attitude, Intentions and Behavior: An Introduction to Theory and Research, Addison Wesley, MA.

FITZGERALD B (2004) A critical look at Open Source. IT Systems Perspectives, Computer - July, 92-94.

FITZGERALD B and KENNY T (2003) Open Source Software in the trenches: lessons from a large-scale OSS implementation. Twenty-Fourth International Conference on Information Systems, 316-326.

FORRESTER CONSULTING (2007) Open Source Software's expanding role in the enterprise. Helping Business Thrive On Technology Change, accessed on-line at 
http://www.unisys.com/eprise/main/admin/corporate/doc/Forrester_researchopen_source_buying_behaviors.pdf(08-June-2008).

GABLE GG (1994) Integrating case study and survey research methods: an example in information systems. European Journal of Information Systems 3(2), 112-126.

GELLINAS R and BIGRAS Y (2004) The characteristics and features of SMEs: favorable or unfavorable logistics integration. Journal of Small Business Management 42(3), 263-278.

GIERA J (2004) The Cost and Risks of Open Source, Helping Business Thrive On Technology Change. Best Practices - Forrester, 2-14.

GLYNN E, Fitzgerald B and EXTON C (2005) Commercial adoption of Open Source Software: an empirical study. Proceedings of International Symposium on Empirical Software Engineering, Noosa Heads, Australia, 255-234.

HEDBERG H, IIVARI N, RAJANEN M and HARJUMAA L (2007) Assuring quality and usability in Open Source Software development. Proceedings of the First International Workshop on Emerging Trends in FLOSS Research and Development, 2-6.

HOLCK J, LARSEN MH and PEDERSEN MK (2004) Identifying business barriers and enablers for the adoption of Open Source Software. ISD 2004, Vilnius, Lithuania, September, working paper 10, 1-15.

HOLCK J, LARSEN MH and PEDERSEN MK (2005) Managerial and technical barriers to the adoption of Open Source Software. Proceedings of the 4th International Conference on COTS Based Software Systems. February, Bilbao Spain, LNCS 34(12), 289-300.

HOUGHTON KA, WINKLHOFER H and ENNEW C (2001) Factors affecting the adoption of the Internet for global marketing in UK exporting SMEs: development of a conceptual model. Paper submitted for consideration: The 30th EMAC Conference, Bergen, Norway. 8-11.May, 1-10.

KSHETRI (2004) Economics of Linux adoption in developing countries: developing with Open Source Software. IEEE Software, 74-81.

KUAN KKY and CHAU PYK (2001) A perception-based model for EDI adoption in small businesses using a technology organization environment framework. Information \& Management 38, 507-521.

KUMAR S and KRISHNAN MS (2005) Impact of Open Source Software adoption on firm IT expenditure. Workshop on Information Systems and Economics, Irvine, California, 1-5.

KWAN SK and WEST J (2005) A conceptual model for enterprise adoption of Open Source Software. In The Standards Edge: Open Season, BOLIN S (Ed), Ann Arbor, Mich: Sheridan Books, 1-14.

LAKHANI KR and von HIPPEL E (2003) How Open Source Software works: 'free' user-to-user assistance. Research Policy 32(6), June 2003, 923-943. 
LARSEN MH, HOLCK J and PEDERSEN MK (2004) The challenges of Open Source Software in IT adoption: enterprise architecture versus total cost of ownership. IRIS'27, Falkenberg, Sweden, August 2004, 2-20, accessed on-line at http://ir.lib.cbs.dk/download/ISBN/x65640946x.pdf (06-Sept-2008).

LIKER JK and SINDI AA (1997) User acceptance of expert systems: a test of the Theory of Reasoned Action. Journal of Engineering and Technology Management 14, 147-173.

LIN L (2006) Impact of users' expertise on the competition between proprietary and Open Source Software. Proceedings of the 39th Annual Hawaii International Conference on System Sciences (HICSS'06), Track 8, $1-10$.

LINCOLN YS and GUBA EG (1985) Naturalistic Inquiry, Beverly Hills, CA: Sage Publications.

MARTIN L (2005) Internet adoption and use in small forms: internal processes, organisational culture and the roles of the owner-manager and key staff. New Technology, Work and Employment 20(3), 190-204.

MAYRING P (2007) On generalization in qualitatively oriented research. Forum: Qualitative Social Research 8(3), Article 26, 1-9, accessed on-line at http://www.qualitativeresearch.net/index.php/fqs/article/view/291/642 (06-Sept-2008).

MEHRTENS J, CRAGG PB and MILLS AM (2001) A model of Internet adoption by SMEs. Information and Management 39, 165-176.

MILES MB and HUBERMAN AM (1994) Qualitative Data Analysis: An Expanded Sourcebook (2nd Edition), Sage, California.

MINDEL JL, MUI L and VERMA S (2007). Open Source Software adoption in ASEAN member countries. Proceedings of the 40th Hawaii International Conference on System Sciences, pp. 1-10.

NETCRAFT (2010) 2010 Web server survey. http://news.netcraft.com/archives/category/web-server-survey/ (23-June-2010).

OVERBY EM, BHARADWAJ AS and BHARADWAJ SG (2006) An investigation of firm-level Open Source Software adoption: theoretical and practical implications. In Open Source Software in Business - Issues and Perspectives (JAIN RK Ed), Hyderabad, India: ICFAI University Press, accessed on-line at http://userwww.service.emory.edu/ eoverby/filesoverby_open_source_adoption_study.pdf (5-May-2008).

PATTON MQ (1999) Enhancing quality and credibility of qualitative analysis. Health Services Research 34(5), Part II, 1189-1208.

ROGERS EM (1995) Diffusion of Innovations (4th Edition), The Free Press, New York.

SANDELOWSKI M (2000) Focus on research methods. Combining qualitative and quantitative sampling, data collection, and analysis techniques in mixed-method studies. Research in Nursing \& Health 23, 246-255.

SHIH Y and FANG K (2004) The use of a Decomposed-Theory of Planned Behavior to study Internet banking in Taiwan. Internet Research 14(3), 213-223. 
SINGH V, TWIDALE MB and RATHI D (2006) Open Source technical support: a look at peer help-giving. Proceedings of the 39th Hawaii International Conference on Systems Sciences, 1-10, accessed on-line at http://csdl.computer.org/comp/proceedings/hicss/2006/2507/06/250760118c.pdf (06-Sept-2008).

TAYLOR M and MURPHY A (2004) SMEs and e-business. Journal of Small Business and Enterprise development 11(3), 280-289.

TAYLOR S and TODD P (1995A) Decomposition and crossover effects in the Theory of Planned Behavior: a study of consumer adoption intentions. International Journal of Research in Marketing 12, 137-155.

TAYLOR S and TODD P (1995B) Understanding information technology usage: a test of competing models. Information Systems Research 6(2), 144-176.

TAYLOR S and TODD P (1995C) Assessing IT usage: the role of prior experience. MIS Quarterly 19(4), 561570.

VALIMAKI M, OKSANEN V. and LAINE J (2005) An empirical look at the problems of Open Source adoption in Finnish municipalities. ICEC' 2005, Aug 15-17. Xi'an China, 514-520.

VENKATESH V and DAVIS FD (2000) A theoretical extension of Technology Acceptance Model: four longitudinal field studies. Management Science 46(2), 186-204.

VENKATESH V, MORRIS MG, DAVIS GB and DAVIS FD (2003) User acceptance of information technology: toward a unified view. MIS Quarterly 27(3), 425-478.

WANG H and WANG C (2001) Open Source Software adoption: a status report. IEEE Software 18(2), 90-95.

WHEELER DA (2007) Why Open Source Software/ Free Software (OSS/FS, FLOSS, or FOSS)? Look at the numbers!, http://www.dwheeler.com/oss_fs_why.html (5-May-2008).

YAMAUCHI Y, YOKOZAWA M, SHINOHARA T. and ISHIDA T (2000) Collaboration with lean media: how open-source software succeeds. CSCW'00, December 2-6, 2000, Philadelphia, PA, 329-338.

YIN RK (2003) Case Study Research: Design and Methods (3rd Edition), Sage Publications, Thousand Oaks, CA. 